University of Texas at El Paso

ScholarWorks@UTEP

$1-2015$

\title{
Why Right-Brain Cultures Are More Flexible: A Possible Explanation of Yu. Manin's Observation
}

Olga Kosheleva

The University of Texas at El Paso, olgak@utep.edu

Vladik Kreinovich

The University of Texas at El Paso, vladik@utep.edu

Follow this and additional works at: https://scholarworks.utep.edu/cs_techrep

Part of the Mathematics Commons

Comments:

Technical Report: UTEP-CS-15-05

Published in International Mathematical Forum, 2015, Vol. 10, No. 4, pp. 175-180.

\section{Recommended Citation}

Kosheleva, Olga and Kreinovich, Vladik, "Why Right-Brain Cultures Are More Flexible: A Possible Explanation of Yu. Manin's Observation" (2015). Departmental Technical Reports (CS). 896.

https://scholarworks.utep.edu/cs_techrep/896

This Article is brought to you for free and open access by the Computer Science at ScholarWorks@UTEP. It has been accepted for inclusion in Departmental Technical Reports (CS) by an authorized administrator of ScholarWorks@UTEP.For more information, please contact Iweber@utep.edu. 


\title{
Why Right-Brain Cultures Are More Flexible: A Possible Explanation of Yu. Manin's Observation
}

\author{
Olga Kosheleva and Vladik Kreinovich \\ University of Texas at El Paso \\ $500 \mathrm{~W}$. University \\ El Paso, TX79968, USA \\ olgak@utep.edu,vladik@utep.edu
}

\begin{abstract}
Yuri Manin, a renowned mathematician, observed that it is much easier for a person raised in a right-brain culture to adjust to the left-brain environment than vice versa. In this paper, we provide a possible explanation for this phenomenon.
\end{abstract}

\section{Manin's Observation: Formulation of the Prob- lem}

Manin's observation: formulation of the problem. In his talk [?], Yuri Manin, a renowned mathematician, made several observations about cultures that place more emphasis on left-brain, logical, discrete reasoning (e.g., traditional Western cultures) and cultures that place more emphasis on right-brain, intuitive, continuous reasoning (e.g., traditional Chinese culture and other Oriental cultures).

One of his observations is that it is much easier for a person raised in the traditional right-brain culture to adopt to a left-brain one than vice versa. For example:

- many students and researchers who are originally from China blossom in the Western academic institutions, but

- for a Western researcher it is much more difficult to fully adjust to a Chinese academic institution.

What we plan to do. In this paper, we provide a possible mathematical explanation for this phenomenon. 


\section{Formalization of the Problem}

Need for a simplified model. To understand the corresponding adjustment phenomena, let us consider the simplest possible mathematical model for such adjustment. For that, we need to formalize what we mean by right-brain (continuous) and left-brain (discrete) cultures, and what we mean by adjustment.

Right-brain (continuous) and left-brain (discrete) cultures: a simplified description. In mathematics, the most natural notion of continuity is the continuity of a function. From this viewpoint, we will model:

- knowledge representations corresponding to right-brain (continuous) cultures by continuous functions, and

- knowledge representations corresponding to left-brain (discrete) cultures by discrete (piece-wise constant) functions.

We need to select finite-parametric families of functions. At any given moment of time, we can only store finitely many parameters, so we have to restrict ourselves to finite-parametric families of functions. How do we select these families?

Functional dependencies $y=f(x)$ are ubiquitous in nature. For example, for a given body:

- the force $y$ is a function of acceleration $x$ (second Newton's law),

- the voltage $y$ is a function of the current $x$ (Ohm's law),

- the acceleration $y$ caused by the Sun's gravity is a function of the distance $x$,

etc. In all these cases, we can observe the values $y_{i}=f\left(x_{i}\right)$ of the desired function $f(x)$ corresponding to different inputs $x_{1}<\ldots<x_{n}$ :

- forces corresponding to different accelerations,

- voltages corresponding to different values of the current, etc.

It is therefore reasonable to consider functions generated by this information, i.e., by finitely many values $x_{1}, \ldots, x_{n}$ and the corresponding values $y_{1}, \ldots, y_{n}$.

Resulting families. In the continuous case, for every two tuples $\left(x_{1}, \ldots, x_{n}\right)$ and $\left(y_{1}, \ldots, y_{n}\right)$, we need to consider a continuous function $f(x)$ for which $f\left(x_{i}\right)=y_{i}$. The simplest such function is a piece-wise linear function that connects the corresponding points, i.e., a function for which:

- $f(x)=y_{1}$ for $x \leq x_{1}$,

- $f(x)=y_{n}$ for $x \geq x_{n}$, and 
- $f(x)=y_{i}+\frac{x-x_{i}}{x_{i+1}-x_{i}}$ when $x_{i} \leq x \leq x_{i+1}$.

In the discrete case, the simplest possible idea is to use a piece-wise constant function whose value $f(x)$ at each point $x$ coincides with the value $y_{i}$ at the nearest point $x_{i}$. In this case,

- $f(x)=y_{1}$ for $x<\frac{x_{1}+x_{2}}{2}$,

- $f(x)=y_{n}$ for $x \geq \frac{x_{n-1}+x_{n}}{2}$, and

- $f(x)=y_{i}$ for $\frac{x_{i-1}+x_{i}}{2} \leq x<\frac{x_{i}+x_{i+1}}{2}$.

What does adjustment mean in these terms? Since we are talking about functions, a natural meaning of adjustment is approximation:

- for a right-brain (continuous) person to adjust to the left-brain (discrete) culture means that we try to approximate a discrete function by continuous ones;

- similarly, for a left-brain (discrete) person to adjust to the right-brain (continuous) culture means that we try to approximate a continuous function by discrete ones.

As a numerical measure of approximation quality, we can use the Least Squares difference - the one that is mostly used in data processing. In our case, this means that, in effect, as a measure of difference between two functions $f(x)$ and $g(x)$ we consider the quantity $\int(f(x)-g(x))^{2} d x$. For this integral to be finite, we need to limit ourselves to a finite interval $[L, U]$.

Now, we are ready to formulate the problem in precise terms.

\section{Definitions and the Main Result}

Definition 1. Let $L<U$ be real numbers, and let $n$ be a positive integer.

- By an n-parametric continuous function, we mean a function that, for some tuples $L \leq x_{1}<x_{2}<\ldots<x_{n} \leq U$ and $\left(y_{1}, \ldots, y_{n}\right)$, has the form

- $f(x)=y_{1}$ for $x \leq x_{1}$,

- $f(x)=y_{n}$ for $x \geq x_{n}$, and

- $f(x)=y_{i}+\frac{x-x_{i}}{x_{i+1}-x_{i}}$ when $x_{i} \leq x \leq x_{i+1}$.

- By an n-parametric discrete function, we mean a function that, for some tuples $L \leq x_{1}<x_{2}<\ldots<x_{n} \leq U$ and $\left(y_{1}, \ldots, y_{n}\right)$, has the form

- $f(x)=y_{1}$ for $x<\frac{x_{1}+x_{2}}{2}$, 


$$
\begin{aligned}
& \text { - } f(x)=y_{n} \text { for } x \geq \frac{x_{n-1}+x_{n}}{2}, \text { and } \\
& \text { - } f(x)=y_{i} \text { for } \frac{x_{i-1}+x_{i}}{2} \leq x<\frac{x_{i}+x_{i+1}}{2} \text {. }
\end{aligned}
$$

Definition 2. By a distance between two functions $f(x)$ and $g(x)$, we mean the value $d(f, g) \stackrel{\text { def }}{=} \int_{L}^{U}(f(x)-g(x))^{2} d x$. For every real number $\varepsilon>0$, we say that $f$ and $g$ are $\varepsilon$-close if $d(f, g) \leq \varepsilon$.

Proposition 1. For every $n$ and for every $\varepsilon>0$, for every $n$-parametric continuous function $f(x)$, there is a $2 n$-parametric discrete function $g(x)$ which is $\varepsilon$-close to $f(x)$.

Proposition 2. For every $n$ and $N>n$, for every non-constant $n$-parametric continuous function $f(x)$, there exists a real value $\varepsilon>0$ such that no $N$ parametric discrete function is $\varepsilon$-close to $f(x)$.

Conclusion. So, while a discrete function can be approximated by continuous functions with any possible accuracy, there is always a limit with which a discrete function can approximate a continuous one. In other words, it is much easier for continuous functions to approximate discrete ones than vice versa, it is much easier for a continuous function to adjust to a discrete family than vice versa. This is exactly what we tried to explain.

\section{Proofs}

Proof of Proposition 1. Let $f(x)$ be a continuous function corresponding to the values $x_{1}<\ldots<x_{n}$ and $y_{1}, \ldots, y_{n}$. To create an approximating discrete function, we approximate each jump by a fast-increasing linear function.

Specifically, let us select a small $\delta>0$, and consider $2(n-1)$ values

$$
x_{1}^{-}<x_{1}^{+}<x_{2}^{-}<x_{2}^{+}<\ldots<x_{n-1}^{-}<x_{n-1}^{+},
$$

where $x_{i}^{-}=\frac{x_{i}+x_{i+1}}{2}-\delta$ and $x_{i}^{+}=\frac{x_{i}+x_{i+1}}{2}+\delta$. As the corresponding $y$-values, we take $y_{i}^{-}=y_{i}$ and $y_{i}^{+}=y_{i+1}$.

One can check that the resulting $2 n$-parametric continuous function $g(x)$ differs from the original discrete function $f(x)$ only on $n-1$ intervals $\left[x_{i}^{-}, x_{i}^{+}\right]$ of length $2 \delta$ each - and thus, of total length $2(n-1) \cdot \delta$. On these intervals, the values of both functions $f(x)$ and $g(x)$ are bounded from below from $y \stackrel{\text { def }}{=}$ $\min \left(y_{1}, \ldots, y_{n}\right)$ and from above by $Y \stackrel{\text { def }}{=} \max \left(y_{1}, \ldots, y_{n}\right)$. Thus,

$$
|f(x)-g(x)| \leq Y-y,
$$

hence $(f(x)-g(x))^{2} \leq(Y-y)^{2}$, and therefore, $d(f, g) \leq(Y-y)^{2} \cdot 2(n-1) \cdot \delta$.

So, when $\delta \rightarrow 0$, we get $d(f, g) \rightarrow 0$. The statement is proven. 
Proof of Proposition 2. Since the $n$-parametric continuous function $f(x)$ is not a constant, there exists an $i$ for which $y_{i} \neq y_{i+1}$. On the corresponding interval $\left[x_{i}, x_{i+1}\right]$, the function $f(x)$ is linear, with a non-zero slope $k \stackrel{\text { def }}{=} \frac{y_{i+1}-y_{i}}{x_{i+1}-x_{i}}$.

Any $N$-parametric discrete function $g(x)$ is based on $N$ pairs $\left(x_{i}^{\prime}, y_{i}^{\prime}\right)$ that divide the whole interval $[L, U]$ into $N$ subintervals on each of which the function $g(x)$ is constant. In particular, some of these intervals cover the whole interval $\left[x_{i}, x_{i+1}\right]$. Thus, the interval $\left[x_{i}, x_{i+1}\right]$ is divided into $\leq N$ parts of each of which $g(x)$ is a constant. The sum of the lengths of these parts is $x_{i+1}-x_{i}$, thus at least one of these parts must have the length $\ell$ which is at least $h \stackrel{\text { def }}{=} \frac{x_{i+1}-x_{i}}{N}$ - else, if all their lengths are smaller, their sum would be smaller than $x_{i+1}-x_{i}$.

On this part of length $\ell$, a linear function $f(x)$ with slope $k$ is approximated by a constant. The distance $f(f, g)=\int_{L}^{U}(f(x)-g(x))^{2} d x$ between $f(x)$ and $g(x)$ is greater than or equal to the integral $\int(f(x)-g(x))^{2} d x$ limited to this part. One can easily check that for a linear function, the smallest $\ell^{2}$-distance from a constant is when this constant is equal to a midpoint between the two extreme points of the linear function. On this part, the corresponding integral is thus equal to

$$
\int_{-\ell / 2}^{\ell / 2}(k \cdot x)^{2} d x=2 \int_{0}^{\ell / 2} k^{2} \cdot x^{2} d x=2 k^{2} \cdot \frac{1}{3} \cdot\left(\frac{\ell}{2}\right)^{3} .
$$

Since the distance $d(f, g)$ is larger than or equal to this integral and $\ell \geq h$, we have

$$
d(f, g) \geq 2 k^{2} \cdot \frac{1}{3} \cdot\left(\frac{h}{2}\right)^{3} .
$$

Any positive real number which is smaller than the right-hand side of this inequality can thus be taken as the desired value $\varepsilon>0$. The proposition is proven.

Acknowledgments. This work was supported in part by the National Science Foundation grants HRD-0734825 and HRD-1242122 (Cyber-ShARE Center of Excellence) and DUE-0926721.

\section{References}

[1] Yu. Manin, "Point, atom, letter", Presentation at the International Interdisciplinary Conference "Philosophy, Mathematics, Linguistics: Aspects of Interaction'2014", St. Petersburg, Russia, April 21-15, 2014. 